\title{
Analysis of COVID-19 patients from Northumbria Healthcare NHS Trust not requiring level 2/3 care
}

\author{
Authors: Avinash Aujayeb ${ }^{\mathrm{A}}$ and Laura Mackay ${ }^{\mathrm{A}}$
}

\section{Introduction}

In Northumbria Healthcare NHS Foundation Trust, the surge of the COVID-19 pandemic occurred in March 2020 and April 2020. It is vital to understand local epidemiological data in the context of national and global data and to delineate any notable differences.

\section{Methods}

A retrospective review of all patients receiving level 1 (ward-based) care with positive SARS-CoV-2 ribonucleic acid PCR on swab between 01 March 2019 and 27 of April 2020 was performed. Local Caldicott approval was granted. Demographics, comorbidities, medication history, presenting symptoms and survival outcomes were collected. Descriptive statistical methodology was applied.

\section{Results}

Three-hundred and forty-five patients (156 female; 189 male) were identified. Mean age was 75 years (range 19-100); $94 \%$ (325 patients) were over the age of 50 years. Mean number of admissions in the preceding 12 months was 1.75 , although 89 patients $(25 \%)$ had no previous admissions during that period. Two-hundred and thirty-two (67\%) were assessed as not suitable for escalation of care or for resuscitation. Overall mortality was $32 \%$. Smoking status was available in 247 patients (72\%): 18 (5\%) were current smokers, 107 (31\%) were ex-smokers, 122 (35\%) were never smokers. One-hundred and seven patients had a background of respiratory disease (66 had chronic obstructive pulmonary disease (COPD), 43 had asthma, 17 had bronchiectasis and one had interstitial lung disease) compared with background hypertension in 144 patients, cardiovascular disease in 113 patients, cerebrovascular disease in 56 patients, dementia in 63 patients, diabetes in 91 patients and chronic kidney disease in 113 patients. Eight patients had haematological malignancies, 44 had solid organ tumours, 10 had chronic liver disease and 10 had inflammatory bowel disease. Only two patients were from Black, Asian and minority ethnic backgrounds; both survived.

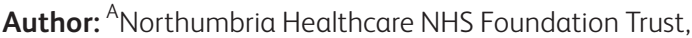
Northumbria, UK

\section{Conclusions}

In patients receiving level 1 care, mortality with SARS-CoV-2 infection was $32 \%$. The majority of patients were elderly, comorbid and frail but survivorship was higher than expected. The patients who received higher level care in our institution have been characterised in a separate audit. Further statistical analysis by age group and by number of comorbidities is under way.

\section{Conflicts of interest}

None declared. 